\title{
ON NORMAL MODES IN CLASSICAL HAMILTONIAN SYSTEMS
}

\author{
E. W. C. VAN GROESEN \\ Mathematical Institute, Catholic University, Toernooiveld, Nijmegen, the Netherlands
}

(Received 16 June 1982)

\begin{abstract}
Normal modes of Hamiltonian systems that are even and of classical type are characterized as the critical points of a normalized kinetic energy functional on level sets of the potential energy functional. With the aid of this constrained variational formulation the existence of at least one family of normal modes is proved and, for a restricted class of potentials, bifurcation of modes is investigated. Furthermore, a conjecture about a lower bound for the number of normal modes in case the potential is homogeneous, is proved.
\end{abstract}

\section{INTRODUCTION}

We consider a $n$-degree of freedom dynamical system with Hamiltonian

$$
H(q, p)=\frac{1}{2}|p|^{2}+V(q)
$$

where the potential $V$ is a continuously differentiable even function that is monotonically increasing on rays through the origin:

(i) $V(q)=V(-q)$ for all $q \in \mathbb{R}^{n}$

(ii) $V(0)=0$ (normalisation)

(iii) for all $q \in \mathbb{R}^{n}$ the function $\rho \rightarrow V(\rho q)$ is monotonically increasing for $\rho>0$.

Because of the specific symmetry of the Hamiltonian, trajectories that pass through the origin are symmetric in $q$-space. In particular, normal modes of the dynamical system [i.e. periodic solutions that pass through the origin and have two "restpoints" (at which the velocity vanishes)] are completely determined by only a part of their trajectory. With this observation it is possible to give a variational characterization of these solutions that differs from the usual characterization with the Jacobi functional.

In fact, in Section 2 we show how normal mode solutions are in one-to-one correspondence with the critical points of the (normalized) total kinetic energy functional, when this functional is constrained to the set of functions that pass through the origin and for which the (normalized) total potential energy has a specified value $(R>0$ say). The period of the normal mode is directly related to the Lagrange multiplier that enters the EulerLagrange equation of this constrained variational problem.

The existence of at least one normal mode (given $R>0$ ) will be proved by showing that the minimal value of this constrained variational problem is actually attained. Hence, varying $R$, a one-parameter family of normal modes is obtained, and the dependence of the period and the energy on the value of $R$ will be investigated. [Using these methods, the same results can also be obtained for more general Hamiltonians of the form

$$
H(q, p)=\frac{1}{2} p \cdot M^{-1}(q) p+V(q),
$$

where $V$ satisfies conditions (1.2), provided that the mass-matrix $M(q): \mathbb{R}^{n} \rightarrow \mathbb{R}^{n}$ satisfies the following conditions:

(i) $q \rightarrow M(q)$ is differentiable for all $q \in \mathbb{R}^{n}$,

(ii) $M(q)=M(-q)$ for all $q \in \mathbb{R}^{n}$,

(iii) $M(q)$ is uniformly positive definite:

$$
M(q)=M^{*}(q) \text { for all } q \in \mathbb{R}^{n},
$$

and there exists $\alpha>0$ such that

$$
\xi \cdot M(q) \xi \geqslant \alpha \mid \xi^{2} \text { for all } \xi \in \mathbb{R}^{n} \text {, all } q \in \mathbb{R}^{n} \text {.] }
$$


In general it is not possible to determine the number of normal modes. However, if the potential $V$ is a homogeneous function, it has been conjectured in the literature, and will be proved in Section 3 , that there are at least $n$ distinct normal modes (with minimal period).

The phenomenon of energy-bifurcation of normal modes in multi-dimensional systems is well known (see e.g. [1]), but in general difficult to investigate in detail. Systems that exhibit this phenomenon, and which can be studied in detail, are obtained if for the potential the "direct" sum of several one-dimensional potentials is taken. For such potentials we shall show in Section 4 that, already for $n=2$, a great variety of bifurcation-phenomena can be observed.

For the results of this paper we use the constrained variational formulation of the normal modes that was mentioned above. In many references, e.g. $[1,2,3]$, the Jacobi functional corresponding to (1.1) is used. This functional is obtained if one observes that solutions of the dynamical system for which the total energy is given ( $E$ say) are stationary points of the constrained action functional

$$
\operatorname{stat}\left\{\int_{0}^{1} p(t) \cdot \dot{q}(\tau) \mathrm{d} \tau \mid H(q(\tau), p(\tau))=E\right\}
$$

Transforming this parameter-independent variational principle in $(q, p)$-space to a variational principle in $q$-space [roughly speaking, for given function $q(\tau)$, maximizing with respect to $p(\tau)]$, the Jacobi-functional corresponding to the Hamiltonian (1.1) is found to be

$$
J(q)=\int_{0}^{1} \sqrt{2(E-V(q)(\tau)))}|q(\tau)| \mathrm{d} \tau,
$$

the stationary points of which are solutions of the dynamical system with the required energy $E$.

The Jacobi-functional has already been used by Seifert [2] to prove the existence of at least one normal mode (without assuming $V$ to be even). Recently, Weinstein [3] has generalized this result to more general, smooth, Hamiltonians assuming only that the set $\left\{(q, p) \in \mathbb{R}^{n} \times \mathbb{R}^{n} \mid H(q, p) \leqslant E\right\}$ is a convex set in $(q, p)$ space surrounding $(0,0)$. Clarke [4] obtains the existence of a periodic solution under the same assumption without requiring that the Hamiltonian is differentiable; he uses methods from convex analysis, in contrast to Seifert and Weinstein who use geometrical methods. Quite different methods have lead Rabinowitz to much the same results [5]; see also Clarke and Ekeland [6]. Under restricted conditions for $H$, lower bounds for the number of periodic solutions have been obtained recently; the interested reader may consult $[7,8,9,10,11]$ and the references therein.

\section{CHARACTERIZATION AND EXISTENCE OF NORMAL MODES}

The Lagrangian corresponding to the Hamiltonian (1.1) is given by

$$
L\left(q, \partial_{t} q\right)=\frac{1}{2}\left|\partial_{t} q\right|^{2}-V(q)
$$

(where $\partial_{t}=\frac{d}{d t}$ ) and the evolution of the system is described by

$$
-\partial_{t}^{2} q(t)=V^{\prime}(q(t))
$$

A normal mode is a periodic solution of (2.2), with (least) period $T$ say, that passes through the origin: $q(0)=q(T)=0$ and for which the velocity vanishes identically (precisely) twice during one period: $\partial_{1} q\left(t_{1}\right)=\partial_{1} q\left(t_{2}\right)=0$ for $0<t_{1}<t_{2}<T$.

From the symmetry of the potential, and applying an appropriate scaling of the timevariable, it follows that normal modes can be obtained as solutions of a (non-linear) eigenvalue problem.

Lemma 2.1. Let $x(\tau) \in \mathbb{R}^{n}, \tau \in[0,1]$, solve for some $\lambda \in \mathbb{R}$ the following boundary value problem:

$$
\begin{aligned}
-\ddot{x}(\tau) & =\lambda V^{\prime}(x(\tau)), \quad \tau \in(0,1) \\
x(0) & =0, \dot{x}(1)=0
\end{aligned}
$$


(where dots denote differentiation with respect to $\tau$ ).

Then $\lambda$ is positive (if $x \neq 0$ ), and the function $q(t), t \in[0, T]$, defined below is a normal mode with period

$$
q(t)=\left[\begin{array}{ll}
\bar{q}(t) & \text { for } 0 \leqslant t \leqslant \frac{1}{2} T \\
-\bar{q}\left(t-\frac{1}{2} T\right) & \text { for } \frac{1}{2} T \leqslant t \leqslant T
\end{array}\right.
$$

where

$$
\bar{q}(t)=\left[\begin{array}{ll}
x(t / \sqrt{ } \lambda) & \text { for } 0 \leqslant t \leqslant \frac{1}{4} T \\
x(2-t / \sqrt{ } \lambda) & \text { for } \frac{1}{4} T \leqslant t \leqslant \frac{1}{2} T
\end{array}\right.
$$

Proof. From condition (1.2.ii) it follows that

$$
V^{\prime}(x) \cdot x>0 \text { for all } x \in \mathbb{R}^{n}, x \neq 0 \text {. }
$$

Taking the $L_{2}$-inner product of the equation for $x(\tau)$ with $x(\tau)$, a partial integration, using the boundary conditions for $x$, shows that $\lambda$ is positive:

$$
\int_{0}^{1}|\dot{x}(\tau)|^{2} \mathrm{~d} \tau=\lambda \int_{0}^{1} V^{\prime}(x(\tau)) \cdot x(\tau) \mathrm{d} \tau
$$

The fact that $q(t)$ is smooth and solves equation (2.2) if $x(\tau)$ solves equation (2.3) is then easily verified. Note in particular that, if $x$ is twice continuously differentiable, the same is true for the function $q$, as $V^{\prime}(0)=0$. Even if $V$ is not differentiable at $x=0$, but has a finite directional derivative in any direction, a solution of (2.3) gives a solution of (2.2) via (2.5) which has at most a finite jump in the component of the second derivative in one direction.

In the following we shall characterize the solutions of (2.3) as the critical points of certain functionals with suitable (boundary- and side-) constraints. A first result in this direction is the well known relation between (2.3) and a version of Hamilton's principle. To express this, let $X$ be the space of functions $x(\tau)$ that are piecewise continuous differentiable and satisfy $x(0)=0$ :

$$
X=\left\{x \in P C^{1}\left([0,1], \mathbb{R}^{n}\right) \mid x(0)=0\right\},
$$

and define the (normalized) action functional [cf. (2.1)]:

$$
\mathscr{L}_{\lambda}(x)=\int_{0}^{1}\left\{\frac{1}{2}|\dot{x}(\tau)|^{2}-\lambda V(x(\tau))\right\} \mathrm{d} \tau
$$

For ease of presentation we shall say that $x$ is a solution of the variational problem

$$
\text { stat }\left\{\mathscr{L}_{\lambda}(x) \mid x \in X\right\}
$$

if $x$ is a critical point of $\mathscr{L}_{\lambda}$ on $X$. In the following we shall frequently use obvious variants of this notation.

Lemma 2.2. A function $x \in C^{2}\left([0,1], \mathbb{R}^{n}\right)$ is a solution of $(2.3)$ if and only if $x$ is a solution of the variational problem (2.9).

Remark 2.3. The boundary condition $\dot{x}(1)=0$ follows as a natural boundary condition from (2.9).

For a restricted class of potentials $V,(2.9)$ can be used to prove the existence of at least one solution of (2.3) as we shall see below.

Another variational characterization of the solutions of (2.3) is obtained if we consider the (normalized) total kinetic energy functional

$$
\mathscr{K}(x):=\int_{0}^{1} \frac{1}{2}|\dot{x}(\tau)|^{2} \mathrm{~d} \tau
$$

on the set of functions that have prescribed (normalized) total potential energy: for $R>0$ let

$$
\mathscr{M}_{R}:=\{x \in X \mid \mathscr{V}(x)=R\}
$$


where

$$
\mathscr{Y}^{\prime}(x):=\int_{0}^{1} V(x(\tau)) \mathrm{d} \tau
$$

If we define $R^{*}>0$ :

$R^{*}:=\left[\begin{array}{ll}\sup \left\{V(x) \mid x \in \mathbb{R}^{n}\right\} & \text { if } V \text { is bounded from above, } \\ \infty & \text { else, }\end{array}\right.$

then $\mathscr{M}_{R}$ is non-empty for $0<R<R^{*}$.

Lemma 2.4. For $R \in\left(0, R^{*}\right)$ let $x$ be a solution of the following constrained variational problem:

$$
\operatorname{stat}\left\{\mathscr{K}(x) \mid x \in \mathscr{M}_{R}\right\} \text {. }
$$

Then there exists some multiplier $\lambda>0$ such that $x$ is a nontrivial (i.e. $x \neq 0$ ) solution of (2.3).

Proof. As $V(x)=0$, and also $V^{\prime}(x)=0$, only if $x=0$, for every $x \in \mathscr{M}_{R}$ it holds $x \neq 0$ and $V^{\prime}(x) \neq 0$. This last property means that $\mathscr{H}_{R}$ is a "regular" manifold in $X$, and the Lagrange multiplier rule can be applied [12]. Consequently, any solution of (2.13) satisfies (2.3) for some $\lambda \in \mathbb{R}$. Then $\lambda>0$ follows from Lemma 2.1 .

The next result states that for any $R \in\left(0, R^{*}\right)$ problem (2.13) has at least one solution.

Theorem 2.5. Let $V$ satisfy the conditions (1.2). Then, for any $R \in\left(0, R^{*}\right)$ there exists a function $x_{R} \in C^{2}\left([0,1], \mathbb{R}^{n}\right)$ that is a solution of the following constrained minimization problem:

$$
\mathscr{P}_{R}: \inf \left\{\mathscr{K}(x) \mid x \in \mathscr{M}_{R}\right\}
$$

This minimal solution $x_{R}$ solves for some $\lambda=\lambda(R)>0$ the boundary value problem (2.3).

Proof. For the proof we use standard Hilbert space methods. Let

$$
\bar{X}:=\left\{x \in H_{1}\left([0,1], \mathbb{R}^{n}\right) \mid x(0)=0\right\},
$$

where $H_{1}\left([0,1], \mathbb{R}^{n}\right)$ is the Sobolev space of continuous $n$-vector functions on $[0,1]$ that have square integrable generalized derivatives. With the inner product

$$
(x, y) \bar{X}:=\int_{0}^{1} \dot{x}(\tau) \cdot \dot{y}(\tau) \mathrm{d} \tau
$$

$\bar{X}$ is a Hilbert space. Furthermore, $\bar{X}$ is not merely continuously, but even compactly embedded in $C^{0}\left([0,1], \mathbb{R}^{n}\right)$ (i.e. any bounded sequence in $X$ has a subsequence that converges in $\left.C^{0}\left([0,1], \mathbb{R}^{n}\right)\right)$. Note that the kinetic energy functional $\mathscr{K}$ is neatly defined on $\bar{X}$, and is in fact half of the square of the norm corresponding to the inner product defined above:

$$
\mathscr{K}(x)=\frac{1}{2}\|x\|_{X}^{2} \quad x \in \bar{X}
$$

Now consider the set

and the minimization problem

$$
\overline{\mathscr{M}}_{R}:=\{x \in \bar{X} \mid \mathscr{V}(x)=R\}
$$

$$
\inf \left\{\mathscr{K}(x) \mid x \in \overline{\mathscr{H}}_{R}\right\} \text {. }
$$

The existence of a solution of (2.16) then follows from a generalization of Weierstrass' theorem [12].

Therefore it suffices to note that the set $\mathscr{M}_{R}$ is closed with respect to the weak topology, and that $\mathscr{K}$ is lower semi-continuous with respect to weak convergence, and coercive (i.e. $\mathscr{K}(x) \rightarrow \infty$ for $\|x\|_{x \rightarrow \infty}$ ). Having shown the existence of a solution $\hat{x}$ of (2.16), the multiplier rule (as in Lemma 2.4) implies that $\hat{x}$ can be interpreted as a generalized solution of (2.3), i.c. $\hat{x} \in \bar{X}$ and for some $\lambda \in R, \hat{x}$ satisfies

$$
\int_{0}^{1} \dot{x}(\tau) \cdot \dot{y}(\tau) \mathrm{d} \tau=\lambda \int_{0}^{1} V^{\prime}(\hat{x}(\tau)) \cdot y(\tau) \mathrm{d} \tau, \quad \text { for all } y \in \bar{X} .
$$


From the well known DuBois-Reymond lemma [12] it follows at once that $\hat{x}$ is in fact at least twice differentiable, and satisfies equation (2.3) in the classical sense.

This proves the theorem.

Remark 2.6. Note that the solution of problem (2.13) is not unique: if $x_{R}$ is a solution then so is $-x_{R}$. But both $x_{R}$ and $-x_{R}$ give rise to the same normal mode (2.5), i.e. the trajectories in $q$-space are the same, but are transferred in opposite directions (time reversal).

In contradistinction to problem (2.9), for the minimization problem (2.13) the value of the parameter $\lambda$ is not prescribed.

In order to find the relation between $R, \lambda(R)$ and the energy of the corresponding normal mode, define the function $h:\left[0, R^{*}\right] \rightarrow \mathbb{R}$ :

$$
h(R):=\inf \left\{\mathscr{K}(x) \mid x \in \mathscr{M}_{R}\right\} .
$$

As for solutions of (2.3) the total energy is conserved, define $E$ by

$$
\left.\frac{1}{2}|\dot{x}(\tau)|^{2}+\lambda V(\tau)\right)=\lambda E, \quad \tau \in[0,1]
$$

(defined in this way, $E$ is precisely the total energy of (2.2) if $q$ and $x$ are related by equation (2.5):

$$
\frac{1}{2}\left|\partial_{t} q(t)\right|^{2}+V(q(t))=E, \quad \text { all } t \in \mathbb{R} \text {.) }
$$

Writing $E=E(R)$ for the solution $x_{R}$ of (2.13), we easily find from (2.18):

Proposition 2.7. For any solution $x_{R}$ of (2.13) the following relations hold:

$$
\begin{aligned}
\lambda(R) \cdot E(R) & =h(R)+\lambda(R) \cdot R \\
E(R) & =V\left(x_{R}(1)\right) .
\end{aligned}
$$

Moreover, the function $\tau \rightarrow V\left(x_{R}(\tau)\right), \tau \in[0,1]$, is maximal at $\tau=1$.

In the rest of this section we shall investigate the function $h$ more thoroughly.

For all potentials $V$ that satisfy conditions (1.2) we have the following basic results:

Proposition 2.8. (i) The function $R \rightarrow h(R), R \in\left[0, R^{*}\right)$ is monotonically increasing with $h(R) \rightarrow \infty$ for $R \uparrow R^{*}$;

(ii) $h$ is continuous on $\left[0, R^{*}\right)$;

(iii) Let $R_{n} \rightarrow \hat{R}$ be any sequence in $\left[0, R^{*}\right)$, and let $x_{n}$ denote a solution of $\mathscr{P}_{R_{n}}$. Then there is a subsequence $\left\{x_{n^{\prime}}\right\}$ and a solution $\hat{x}$ of $\mathscr{P}_{R^{\prime}}$ such that $\left\{x_{n}\right\}$ converges strongly to $\hat{x}$ in $X$ : i.e.

and

$$
\begin{aligned}
& \left(\mathscr{X}\left(x_{n^{\prime}}\right) \rightarrow \mathscr{X}(\hat{x}), \quad\right. \text { by (ii)) } \\
& x_{n^{\prime}} \rightarrow \hat{x} \text { in } C^{0}\left([0,1], \mathbb{R}^{n}\right)
\end{aligned}
$$

$$
\mathscr{H}\left(x_{n^{\prime}}-\hat{x}\right) \rightarrow 0, \text { for } n^{\prime} \rightarrow \infty .
$$

Proof. (i) The monotonicity follows at once from the observation that any solution of (2.13) is also a solution of

$$
\inf \{\mathscr{K}(x) \mid x \in X, \mathscr{V}(x) \geqslant R\} .
$$

Indeed, if $\hat{x}$ were a solution of (2.21) with $\mathscr{V}(x)>R$, then $\hat{x}$ would be a stationary point of $\mathscr{X}$ on $X$, and must therefore vanish identically: $\hat{x} \equiv 0$, contradicting the property $\mathscr{V}(\hat{x})>R \geqslant 0$.

To show that $h$ is unbounded, suppose that $h$ is bounded for $R \uparrow R^{*}$. Take any sequence $R_{n} \uparrow R^{*}$, and let $x_{n}$ be a solution of $\mathscr{P}_{R_{n}}$. This sequence $\left\{x_{n}\right\}$ is uniformly bounded in $X$ (by assumption), and from the compactness of the embedding in $C^{0}$, it follows that some subsequence $\left\{x_{n^{\prime}}\right\}$ converges in $C^{0}$ to some $\hat{x} \in \bar{X}$ (cf. proof of Theorem 2.5). But then $\mathscr{V}\left(x_{n^{\prime}}\right) \rightarrow \mathscr{V}(\hat{x})$, and thus $\mathscr{V}(\hat{x})=R^{*}$, which is not possible in view of the definition of $R^{*}$ and property 1.2(iii) of the potential $V$. Thus $h(R) \rightarrow \infty$ for $R \uparrow R^{*}$.

(ii) The proof of the continuity of the function $h$ is more involved. To prove that $h$ is continuous at $\hat{R} \in\left[0, R^{*}\right)$ it suffices to show that $h\left(R_{n}\right) \rightarrow h(\hat{R})$ for any monotone sequence $R_{n} \rightarrow \hat{R}$ (decreasing if $\hat{R}=0$ ). Let $R_{n}$ be such a monotone sequence, and $x_{n}$ a solution of 
$\mathscr{P}_{R_{n}}$. Then, as above, $\left\{x_{n}\right\}$ has a subsequence $\left\{x_{n^{n}}\right\}$ that converges weakly in $X$ (as $\left\|x_{n}\right\|_{X}$ is uniformly bounded), and strongly in $C^{0}: x_{n^{\prime}}-\bar{x}$ in $\bar{X}$ and $x_{n^{\prime}} \rightarrow \bar{x}$ in $C^{0}$. From this it follows that $\mathscr{V}^{\prime}(\bar{x})=\hat{R}$, thus $\bar{x} \in \mathscr{M}_{R}$ and by the weak lower semi-continuity of $\mathscr{K}$ :

$$
\mathscr{K}(\bar{x}) \leqslant \lim \inf \mathscr{K}\left(x_{n^{\prime}}\right) \text {. }
$$

From (i), $\mathscr{K}\left(x_{n}\right)=h\left(R_{n}\right)$ is a monotone, bounded sequence, and thus

$$
\mathscr{K}(\bar{x}) \leqslant \lim h\left(R_{n}\right)
$$

As $\bar{x} \in \overline{\mathscr{M}}_{R}$ the definition of $h$ implies:

$$
h(\hat{R}) \leqslant \mathscr{K}(\bar{x}) \leqslant \lim h\left(R_{n}\right) .
$$

(This shows that $h$ is lower semi-continuous.)

Now suppose that

$$
h(\hat{R})=\lim h\left(R_{n}\right)-\varepsilon,
$$

for some $\varepsilon>0$. With $\hat{x}$ a solution of $\mathscr{P}_{\hat{R}}$ let $\rho_{n}>0$ be such that $\mathscr{V}\left(\rho_{n} \hat{x}\right)=R_{n}$. From $R_{n} \rightarrow \hat{R}$ it follows that $\rho_{n} \rightarrow 1$, and thus $\rho_{n} \hat{x} \rightarrow \hat{x}$ strongly in $\hat{X}$. Consequently, $\mathscr{K}\left(\rho_{n} \hat{x}\right) \rightarrow \mathscr{X}(\hat{x})$, which contradicts the assumption (2.23). Hence: $h(\hat{R})=\lim h\left(R_{n}\right)$, which was to be proved.

(iii) This part of the proof is already almost contained in the proof of (ii): from the continuity of $h$, and (2.22) it follows that $\mathscr{K}(\bar{x})=h(\hat{R})$, such that, as $\bar{x} \in \mathscr{M}_{R}, \bar{x}$ is a solution of $\mathscr{P}_{\hat{R}}$. For the subsequence $\left\{x_{n^{\prime}}\right\}$ we then have:

$$
x_{n^{\prime}} \rightarrow \bar{x} \text { in } \bar{X} \text {, and }\left\|x_{n^{\prime}}\right\|_{\bar{X}} \rightarrow\|\bar{x}\|_{\bar{X}}
$$

From this it follows that $x_{n}$, converges strongly in $\bar{X}$ :

$$
\left\|x_{n} \cdot-\bar{x}\right\|_{\bar{x}} \rightarrow 0 \text {. }
$$

Remark 2.9. From the monotonicity of the function $h$ it can be shown, using methods from [13], that the minimization problem (2.13) is in a one-to-one correspondence with an inverse maximization problem, i.e. for any $R \in\left(0, R^{*}\right)$ there exists an unique $r=r(R)>0$ such that the solutions of $\mathscr{P}_{R}$ are also solution of

$$
\sup \{\mathscr{V}(x) \mid x \in X, \mathscr{K}(x)=r\} \quad(r>0),
$$

and conversely.

As for any monotone function, the function $h$ is differentiable almost everywhere on $\left[0, R^{*}\right]$. If it exists, its derivative is precisely the multiplier $\lambda(R)$ :

Proposition 2.10. (i) If $h_{+}^{\prime}(R),\left(h_{-}^{\prime}(R)\right)$ denotes the right (left) hand side derivative of the function $h$ at $R$, then we have

$$
h_{+}^{\prime}(R) \leqslant \lambda(R) \leqslant h_{-}^{\prime}(R), \quad R \in\left(0, R^{*}\right) .
$$

(ii) If $h$ is differentiable at $R$, then

$$
\lambda(R)=h^{\prime}(R)=\frac{\mathrm{d}}{\mathrm{d} R} h(R) .
$$

Proof. Of course, (ii) is a simple consequence of (i). Property (i) has been proved in [13]. Let us give a direct proof of (ii) under the extra assumption that for each $R$ it is possible to take a solution $x_{R}$ of $\mathscr{P}_{R}$ such that the mapping $R \rightarrow x_{R}$ is differentiable. In that case, writing $y(\tau)=\mathrm{d} x_{R}(\tau) / \mathrm{d} R$, the function $y(\tau)$ satisfies

$$
y(0)=0, \quad \dot{y}(1)=0 \quad \text { and } \quad \int_{0}^{1} V^{\prime}\left(x_{R}(\tau)\right) \cdot y(\tau) \mathrm{d} \tau=1 .
$$

The result then follows in a straightforward way:

$$
\begin{aligned}
\frac{\mathrm{d}}{\mathrm{d} R} h(R) & =\frac{\mathrm{d}}{\mathrm{d} R} \int_{0}^{1} \frac{1}{2}\left|\dot{x}_{R}(\tau)\right|^{2} \mathrm{~d} \tau \\
& =\int_{0}^{1} \dot{x}_{R}(\tau) \cdot \dot{y}(\tau) \mathrm{d} \tau
\end{aligned}
$$




$$
\begin{aligned}
& =-\int_{0}^{1} \ddot{x}_{R}(\tau) \cdot y(\tau) \mathrm{d} \tau \quad \text { (partial integration) } \\
& \left.=\lambda(R) \int_{0}^{1} V^{\prime}\left(x_{R}(\tau)\right) \cdot y(\tau) \mathrm{d} \tau \quad \text { (equation for } x_{R}\right) \\
& =\lambda(R) .
\end{aligned}
$$

From Lemma 2.2 and Theorem 2.5 it follows that any solution of (2.13) is also a critical point of the functional $\mathscr{L}_{\lambda(R)}$ on $X$. The behaviour of the function $h$ in a neighbourhood of $R$ (in fact, the second derivative $h^{\prime \prime}(R)$ if it exists) determines the character of this critical point.

Proposition 2.11. (i) The function $h$ is locally convex in a neighbourhood of the point $R \in\left(0, R^{*}\right)$ iff any solution $x_{R}$ of $\mathscr{P}_{R}$ (with multiplier $\lambda$ ) is a local minimal point of the functional $\mathscr{L}_{\lambda}$ on $x$.

(ii) The function $h$ is convex for all $R \in\left(0, R^{*}\right)$, iff for all $R$, any solution $x_{R}$ of $\mathscr{P}_{R}$ (with multiplier $\lambda$ ) is a global minimal point of $\mathscr{L}_{\lambda}$ on $X$, i.e. iff $x_{R}$ is a solution of

$$
\inf \left\{\mathscr{L}_{\lambda}(x) \mid x \in X\right\}
$$

Proof

If the function $R \rightarrow h(R)$ is differentiable at $\bar{R} \in\left(0, R^{*}\right)$, the tangent of its graph at $\bar{R}$ is given by

$$
R \rightarrow h(\bar{R})+\lambda(\bar{R}) \cdot(R-\bar{R}) .
$$

First suppose that $h$ is locally convex at $\bar{R}$ (then $h$ is differentiable at $\bar{R}$ as follows from proposition 2.10 (i)). Then, for $|R-\bar{R}|$ sufficiently small, we have

$$
h(R) \geqslant h(\bar{R})+\lambda(\bar{R}) \cdot(R-\bar{R}),
$$

thus

$$
h(R)-\lambda(\bar{R}) R \geqslant h(\bar{R})-\lambda(\bar{R}) \bar{R} .
$$

Hence, with $\bar{x}$ any solution of $\mathscr{P}_{R}^{-}$,

$$
\inf \left\{\mathscr{L}_{\lambda(\bar{R})}(x) \mid x \in \mathscr{M}_{R}\right\} \geqslant \mathscr{L}_{\lambda(\bar{R})}(\bar{x})
$$

for all $R$ for which $|R-\bar{R}|$ is sufficiently small.

From this it follows that

$$
\mathscr{L}_{\lambda(\bar{R})}(x) \geqslant \mathscr{L}_{\lambda(\bar{R})}(\bar{x})
$$

for all $x \in X$ that are in a sufficiently small weak (i.e. $C^{\circ}$-) neighbourhood of $\bar{x}$. This means that $\bar{x}$ is a local minimal point of $\mathscr{L}_{\lambda(\bar{R})}$. If $h$ is convex for all $R \in\left(0, R^{*}\right)$, then (2.28) holds for all $R \in\left(0, R^{*}\right)$, and consequently (2.29) for all $x \in X: \bar{x}$ is a global minimal point of $\mathscr{L}_{\lambda(\bar{R})}$ on $X$.

On the other hand, if $\bar{x}$ is a global minimal point of $\mathscr{L}_{\lambda(\bar{R})}$ on $X$, then

$$
h(\bar{R})-\lambda(\bar{R}) \cdot \bar{R}=\mathscr{L}_{\lambda(\bar{R})}(\bar{x}) \leqslant \mathscr{K}(x)-\lambda(\bar{R}) \mathscr{Y}(x)
$$

for all $x$.

In particular this implies that for all $R$ inequality $(2.30)$ holds for all $x \in \mathscr{M}_{R}$. Hence it follows that

$$
h(\bar{R})-\lambda(\bar{R}) \cdot \bar{R} \leqslant h(R)-\lambda(\bar{R}) \cdot R
$$

for all $R$, i.e. $h$ is convex at $\bar{R}$.

Finally, suppose that $h$ is not locally convex at $\bar{R}$. Then, for any $\lambda \in \mathbb{R}$ it is possible to 
find a sequence $R_{n} \rightarrow \bar{R}$ such that

$$
h\left(R_{n}\right)<h(\bar{R})+\lambda\left(R-R_{n}\right) .
$$

Let $x_{n}$ be a solution of $\mathscr{P}_{\boldsymbol{R}_{n}}$, and $\lambda_{n}$ the corresponding multiplier. Then (proposition 2.8.) for some subsequence $\left\{x_{n^{\prime}}\right\}: x_{n^{\prime}} \rightarrow \bar{x}, \lambda_{n^{\prime}} \rightarrow \bar{\lambda}$, where $\bar{x}$ is a solution of $\mathscr{P}_{R}^{-}$, with $\bar{\lambda}$ the corresponding multiplier. Hence:

$$
\mathscr{K}\left(x_{n^{\prime}}\right)<\mathscr{K}(\bar{x})+\bar{\lambda}\left(\mathscr{V}(\bar{x})-\mathscr{V}\left(x_{n^{\prime}}\right)\right)
$$

from which it follows that $\bar{x}$ is not a local minimal point of the functional $\mathscr{L}_{\lambda}^{-}$on $x$. This completes the proof.

To conclude this section we state two simple criteria for the potential $V$ from which one can deduce that $h$ is globally concave, resp. globally convex. These results will be used in Section 4 where specific examples will also be given.

Lemma 2.12. Suppose that for $V \in C^{2}\left(\mathbb{R}^{n}, \mathbb{R}\right), R^{*}=\infty$, and that $V$ satisfies, besides conditions (1.2),

$$
V^{\prime}(x) \cdot x-V^{\prime \prime}(x) x \cdot x<0 \text { for all } x \in \mathbb{R}^{n}, x \neq 0
$$

Then $R \rightarrow h(R)$ is a strictly concave function, and $R \rightarrow \lambda(R)$ is monotonically decreasing.

Proof. For $\bar{R}>0$, let $\bar{x}$ be any solution of $\mathscr{P}_{\bar{R}}^{-}$and $\bar{\lambda}$ the corresponding multiplier. We shall show that for all $R$, with $0<|R-\bar{R}|$ sufficiently small:

$$
h(R)<h(\bar{R})+\bar{\lambda}(R-\bar{R})
$$

which will prove the statement. To that end consider the function:

$$
\rho \rightarrow \mathscr{K}(\rho \bar{x})-\bar{\lambda}(\rho \bar{x})=: f(\rho)
$$

in a neighbourhood of $\rho=1$. Then $\frac{\mathrm{d} f}{\mathrm{~d} \rho}(1)=0$, and

$$
\begin{aligned}
\frac{\mathrm{d}^{2} f}{\mathrm{~d} \rho^{2}}(1) & =\int_{0}^{1}\left[\dot{\bar{x}}^{2}-\lambda V^{\prime \prime}(\bar{x}) \cdot \bar{x} \cdot \bar{x}\right] \mathrm{d} t \\
& =\lambda \int_{0}^{1}\left[V^{\prime}(\bar{x}) \cdot \bar{x}-V^{\prime \prime}(\bar{x}) \cdot \bar{x} \cdot \bar{x}\right] \mathrm{d} t .
\end{aligned}
$$

With (2.3.2) it follows that $\frac{\mathrm{d}^{2} f}{\mathrm{~d} \rho^{2}}(1)<0$, i.e. that $f$ has a local maximum at $\rho=1$. Consequently:

$$
\mathscr{K}(\rho \bar{x})-\bar{\lambda} \mathscr{V}(\rho \bar{x})<\mathscr{K}(\bar{x})-\overline{\lambda \mathscr{V}}(\bar{x})
$$

for all $\rho$, with $0<|\rho-1|$ sufficiently small. As to any $R$, with $|R-\bar{R}|$ sufficiently small there corresponds a unique $\rho(R)$ in a neighbourhood of 1 such that $\mathscr{V}(\rho(R) \bar{x})=R$ and because $\mathscr{K}(\rho(R) \bar{x}) \geqslant h(R)$ by definition of $h$, inequality (2.33) follows.

Corollary 2.13. In the situation of Lemma 2.12 there is a one-to-one correspondence between $R$ and $E(R)$ : the function $R \rightarrow E(R)$ is monotonically increasing for all $R>0$.

Proof. This is immediate from (2.19) and the monotonicity of the functions $h(R)$ and $\lambda(R)$.

It seems much more difficult to state a general condition that ensures that $h$ is strictly convex. However, if $n=1$ we have the following simple result.

Lemma 2.14. Let $n=1$, and suppose that the potential $V$ satisfies (1.2) together with

$$
V^{\prime}(x) \cdot x-V^{\prime \prime}(x) \cdot x^{2}>0 \text { for all } x \in \mathbb{R}, x \neq 0
$$


Then the function $R \rightarrow h(R)$ is strictly convex (and hence differentiable) on $\left(0, R^{*}\right)$, and $R \rightarrow \lambda(R)$ is monotonically increasing. If $R^{*}$ is finite, then $\lambda(R) \rightarrow \infty$ for $R \rightarrow R^{*}$.

Proof. Let $x$ be any solution of $\mathscr{P}_{R}$; then $x$ is sign definite $(0,1)$, positive say (c.f. also Section 4). As the equation for $x$ can be written as

$$
-\ddot{x}-\lambda V^{\prime \prime}(x) \cdot x=\lambda\left(V^{\prime}(x)-V^{\prime \prime}(x) \cdot x\right)
$$

the function $v \equiv x$ satisfies:

$$
\begin{gathered}
-\ddot{v}-\lambda V^{\prime \prime}(x) v>0 \text { on }(0,1) \\
v(0)=\dot{v}(1)=0 \\
v>0 \text { on }(0,1) .
\end{gathered}
$$

This implies that the lowest eigenvalue $\sigma_{1}$ of the eigenvalue problem:

$$
\begin{gathered}
-\ddot{\psi}-\lambda V^{\prime \prime}(x) \psi=\sigma \psi \\
\psi(0)=\dot{\psi}(1)=0
\end{gathered}
$$

is strictly positive: $\sigma_{1}>0$. Consequently, the second variation of the functional $\mathscr{L}_{\lambda}$ at $x$ in any direction $y$ is strictly positive:

$$
\int_{0}^{1}\left(\dot{y}^{2}-\lambda V^{\prime \prime}(x) y^{2}\right) \mathrm{d} t \geqslant \sigma_{1} \int_{0}^{1} y^{2} \mathrm{~d} t \quad \forall y \subseteq X
$$

which shows that $x$ is a strict local minimal point of the functional $\mathscr{L}_{\lambda}$ on $X$. With proposition 2.11 it follows that $h$ is locally convex at any $R \in\left(0, R^{*}\right)$. Hence $h$ is convex on $\left(0, R^{*}\right)$, and $R \rightarrow \lambda(R)$ is nondecreasing. To show that $h$ is strictly convex, i.e. that $\lambda$ is monotonically increasing with $R$, suppose, on the contrary that $\lambda(R)=\lambda$ for all $R \in\left[R_{1}, R_{2}\right] \subset\left(0, R^{*}\right)$. Then, every solution $x_{R}$ of $\mathscr{P}_{R}$, with $R \in\left[R_{1}, R_{2}\right]$ satisfies the same

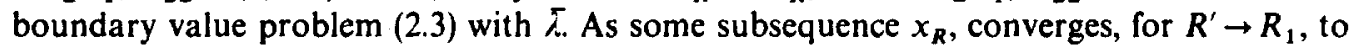
a solution $x_{1}$ of $\mathscr{P P}_{R_{1}}, x_{1}$ is not an isolated solution of (2.3), which contradicts the fact that $\sigma_{1}>0$. As $h(R) \rightarrow \infty$ for $R \uparrow R^{*}$, it is clear that $\lambda(R) \uparrow \infty$ for $R \uparrow R^{*}$ if $R^{*}$ is finite.

\section{HOMOGENEOUS POTENTIALS}

In this section we shall consider potentials that are even and homogeneous functions of degree $k$, with $k>1$ :

$$
V(\sigma x)=\sigma^{k} V(x) \text { for all } x \in \mathbb{R}^{n}, \sigma>0 .
$$

(Note that in general $V$ is not differentiable at the origin.)

In this special case it is a simple matter to determine in an explicit way the dependence of the solution of (2.3) on the parameter $R$ as introduced in the last part of the foregoing section. Just to demonstrate some of the results obtained there we list the relevant relations for this specific example.

Proposition 3.1. Suppose $x_{1}$ is a solution of (2.3) with $\lambda=\lambda(1)$ for which

$$
\int_{0}^{1} V\left(x_{1}\right) \mathrm{d} \tau=1
$$

Then $x_{R}:=R^{1 / k} x_{1}$ is a solution of (2.3) with $\lambda=\lambda(R)=\lambda(1) . R^{(2-k) / k}$, for which

$$
\int_{0}^{1} V\left(x_{R}\right) \mathrm{d} \tau=R
$$

the total energy of this solution, $E(R)$ defined by (2.13), is given by

$$
E(R)=\left(1+\frac{1}{2} k\right) R,
$$


and, furthermore,

$$
\int_{0}^{1} \dot{x}_{R}^{2} \mathrm{~d} \tau=R^{2 / k} \int_{0}^{1} \dot{x}_{1}^{2} \mathrm{~d} \tau
$$

In particular, for the solution of (2.3) that is also a solution of $\mathscr{P}_{R}$, we have

$$
\begin{aligned}
& h(R)=h(1) \cdot R^{2 / k} \\
& \text { and } \lambda(R)=h(1) \cdot \frac{2}{k} \cdot R^{(2-k) / k}=\lambda(1) \cdot R^{(2-k) / k}
\end{aligned}
$$

Note that (3.6) agrees with (2.19) and the convexity or concavity of the function $h$, for $k<2$ resp. $k>2$, agrees with Lemmas 2.12 and 2.14 .

For homogeneous potentials it is possible to find a lower bound on the number of normal modes. It is well known that if $n=2$ there are at least two different normal modes [14]. The following result is generally accepted for granted [15], but as I have not been able to find a reference in the literature, a proof will be given.

Theorem 3.2. Suppose $V$ is an even, homogeneous function of degree $k>1$. Then, for any $E>0$, there are at least $n$ distinct normal modes of (2.1) with total energy $E$, whose trajectories lie on straight lines through the origin.

Remark 3.3. Simple examples show that the lower bound $n$ is the best possible, and that the number of normal modes is not "stable" for small perturbations of the potential.

[Take $V_{\varepsilon}(x)=x-A_{\varepsilon} x$, where for $\varepsilon \in \mathbb{R}, A_{\varepsilon}: \mathbb{R}^{n} \rightarrow \mathbb{R}^{n}$ is a family of symmetric matrices which have $n$ distinct eigenvalues only if $\varepsilon \neq 0$.]

Proof of Theorem 3.2. We shall look for solutions of equation (2.3) that can be written as

$$
x(t)=\alpha(t) e,
$$

where $\alpha$ is a scalar function and $e \in \mathbb{R}^{n}$ is fixed (normalized so that $|e|=1$ ). Inserting relation (3.7) into equation (2.3) it follows [using $V^{\prime}(\sigma x)=\sigma^{k-1} V^{\prime}(x)$, valid for all $x \in \mathbb{R}^{n}, \sigma>0$, ] that (3.7) is a solution if and only if the following two conditions are satisfied:

(i) $e \in \mathbb{R}^{n}$ satisfies for some $\sigma \in \mathbb{R}$

$$
V^{\prime}(e)=\sigma e,|e|=1,
$$

(ii) the function $\alpha$ satisfies for some $\lambda>0$

$$
\begin{aligned}
& -\ddot{\alpha}=\sigma \lambda \alpha^{k-1} \\
& \alpha(0)=0 ; \dot{\alpha}(1)=0 ; \alpha(\tau)>0 \text { for } 0<\tau \leqslant 1 .
\end{aligned}
$$

[The condition $|e|=1$ is just a matter of normalization: any other normalization of $e$ will, in general, change the value of $\sigma$ and the function $\alpha$ by a multiplicative factor without changing the function $x$ as given by relation (3.7) or the value of $\lambda$ (nor the value of $\left.\left.\int_{0}^{1} V(\alpha(\tau) e) \mathrm{d} \tau\right) \cdot\right]$

Let us first concentrate on condition (i), the (non-linear) eigenvalue problem which causes most trouble in the literature on normal modes. The following theorem will give the required result.

Theorem (Ljusternik, 1930). Let $f$ be a function defined on the 1-sphere $S$ in $\mathbb{R}^{n}$ with $f \in C^{1}(S$, $\mathbb{R})$. Assume that $f$ is even: $f(x)=f(-x) \forall x \in S$. Then $f$ has at least $n$ pair of critical points on $S$, i.e. there are $x_{i} \in S, i=1, \ldots, n$, with $x_{i} \neq x_{j}$, and numbers $\sigma_{i} \in \mathbb{R}, i=1, \ldots, n$, such that

[and

$$
f^{\prime}\left(x_{i}\right)=\sigma_{i} x_{i} \quad\left(\sigma_{i}=f^{\prime}\left(x_{i}\right) \cdot x_{i}\right)
$$

Remark. For a convenient reference see [16] or [17]. Simple examples show that the evenness of the function $f$ is essential. This result is well known if $n=2$, and for $n>2$, if $f$ is quadratic: if $n=2$ the maximal and the minimal points of $f$ together are two pairs of critical points, and if $f$ is quadratic [whence $(\cdot)$ is a linear eigenvalue problem] it is a 
consequence of the fact that any symmetric matrix in $\mathbb{R}^{n}$ has a complete set of eigenvectors In the quadratic case the critical values [i.e. $f\left(x_{i}\right)$ ] can be obtained with the usual maxi-mini characterizations of Poincare or Courant-Weyl. In the general case such maxi-mini characterizations can also be obtained. (Using topological properties of a manifold, in this case the sphere $S$, to obtain a lower bound for the number of critical points of a function defined on it is known as the "Ljusternik-Schnirelmann critical point theory".)

From Ljusternik's theorem it follows that (3.8) has at least $n$ pair distinct solutions $\pm e_{i}$; the corresponding eigenvalues $\sigma_{i}$ are positive, as follows with Euler's identity $\left(V^{\prime}(x) \cdot x=k V(x), \forall x\right)$ :

$$
\sigma_{i}=k V\left(e_{i}\right)
$$

The existence of a function $\alpha$ which satisfies (3.9) can then be deduced in several ways. One way is to note that, for given $R>0[E$ and $R$ related by (3.3)], a solution of the form (3.7) with $\int_{0}^{1} V(x) \mathrm{d} \tau=R$ and $\alpha \geqslant 0$ satisfies $\int \alpha^{k}(\tau) \mathrm{d} \tau=R / V(e)$, and the function which solves equation (3.9) is just the (positive) solution of the minimization problem

$$
\inf \left\{\left.\int_{0}^{1} \dot{\alpha}^{2} \mathrm{~d} \tau\left|\int_{0}^{1}\right| \alpha\right|^{k} \mathrm{~d} \tau=R / V(e), \alpha(0)=0\right\}
$$

(this is a special case of problem (2.16) for scalar functions).

Another way is to observe that the solution of equation (3.9) can be obtained explicitly [given $i \sigma>0$, the value of $E$ (or $R$ ) follows]: the solution of equation (3.9) is related to inverse $\beta$-functions (cf. [18]).

Resuming, we have proved the existence of at least $n$ pairs of solutions of equation (2.3) of the form of relation (3.7). These solutions give rise to $n$ distinct normal modes solutions of (2.1), whose trajectories lie on straight lines through the origin, these lines being determined by the eigenvectors $e_{i}$ of (3.8).

\section{BIFURCATION OF NORMAL MODES}

In this section we give some examples of dynamic systems for which the normal modes can be found more or less explicitly. In particular we shall investigate the phenomenon of energy-bifurcation of normal modes: the abrupt appearance or disappearance of normal modes when some parameter (related to the energy) is varied.

To that end we shall consider $n$-dimensional systems with a potential that is the "direct" sum of several one-dimensional potentials, i.e.

$$
V(x)=\sum_{i=1}^{n} V_{i}\left(x_{i}\right), \quad x=\left(x_{i}, \ldots, x_{n}\right)
$$

where each function $V_{i}: \mathbb{R} \rightarrow \mathbb{R}$ satisfies condition (1.2). (Systems that can be written in this way, possibly after a suitable transformation, have been called "splittable systems" in [19]; see this reference for examples.) For completeness let us first briefly investigate the relevant properties of some characteristic one-dimensional systems.

\section{One-dimensional systems}

If $n=1$, the dynamics are described by the scalar second order equation (2.2). In order to find the solution, first note that the particular solution of equation (2.3), which is the minimal solution of problem (2.13), is sign-definite (positive say) and then monotonically increasing and concave. [This sign-definiteness follows from the observation that if $\dot{x}$ solves problem (2.13), then so does $|\hat{x}|$. Therefore $|\hat{x}|$ is a smooth solution of equation (2.3), and the unique solvability of the initial value problem of the equation in (2.3) implies that $|\hat{x}|$ vanishes identically on $[0,1]$ if it vanishes for some $\tau \in(0,1)$. The monotonicity and the concavity of the positive solution is a consequence of the fact that the second derivative has constant sign (negative).]

This minimal solution can be obtained along well known lines. To that end it is simplest 
to take the maximum value of the positive solution as a parameter:

$$
\alpha:=x(1)=\max _{\tau \in[0,1]} x(\tau)
$$

(c.f. proposition 2.7), and express all other quantities in terms of this parameter.

From energy conservation:

$$
\frac{1}{2}|\dot{x}(\tau)|^{2}+\lambda V(x(\tau))=\lambda V(\alpha), 0 \leqslant \tau \leqslant 1,
$$

the solution is easily found in an implicit way:

$$
\tau=\int_{0}^{x(\tau)}\{2 \lambda(\alpha) \cdot[V(\alpha)-V(\xi)]\}^{-\frac{1}{2}} \mathrm{~d} \xi, \tau \in[0,1],
$$

where $\lambda=\lambda(\alpha)$ is given by:

$$
\sqrt{ }(2 \lambda(\alpha))=\int_{0}^{\alpha}[V(\alpha)-V(\xi)]^{-\frac{1}{2}} \mathrm{~d} \xi .
$$

The parameter $R$ introduced before can be expressed as a function of $\alpha$ in the following way: as

$$
R=\int_{0}^{1} V(x(\tau)) \mathrm{d} \tau=\int_{0}^{x} V(x)\left(\frac{\mathrm{d} \tau}{\mathrm{d} x}\right) \cdot \mathrm{d} x
$$

it follows that

$$
R(\alpha)=\frac{1}{\sqrt{(2 \lambda(\alpha))}} \int_{0}^{\alpha} V(x) \cdot[V(\alpha)-V(x)]^{-\frac{1}{2}} \mathrm{~d} x .
$$

The function $\alpha \rightarrow h(R(\alpha))$ can then be found from (2.19) and (2.20):

$$
h(R(\alpha))=\lambda(\alpha) \cdot[\mathrm{V}(\alpha)-\mathrm{R}(\alpha)]
$$

Let us now consider some specific (but characteristic) examples. In view of the applications we have in mind, we are especially interested in the relation between $R$ and $\lambda$.

Example 4.1. $V_{1}(x)=\frac{1}{2} x^{2}$. In this case the equation is linear; the explicit solution is given by

$$
x(\tau)=\alpha \sin \pi / 2 \cdot \tau
$$

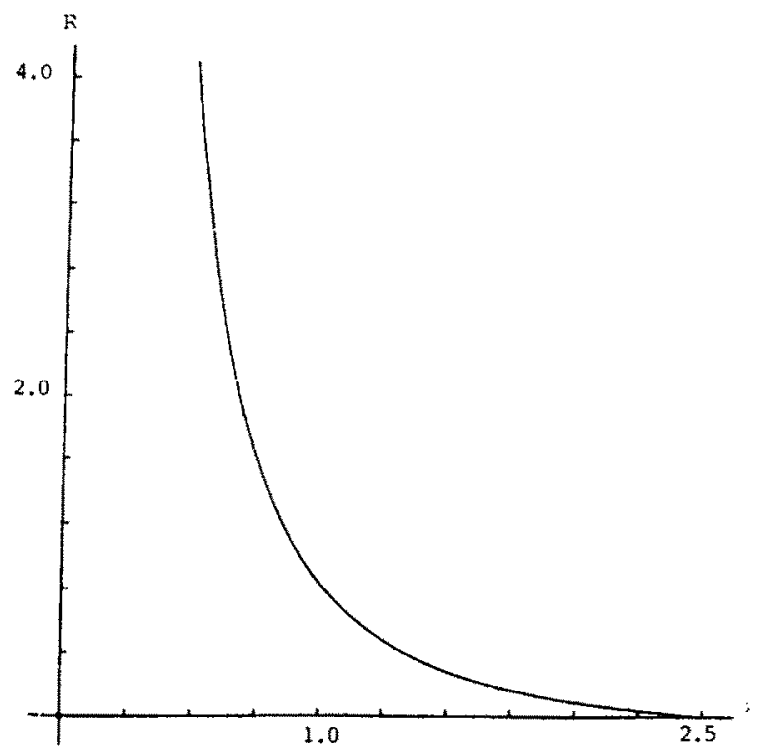

Fig. 1. Graphs of $R$ vs $\lambda$ for the potential $V_{i,} i=3,4$ and 5 respectively, from Examples $4.3-4.5$. These figures have been obtained with numerical means from Equations (4.5) and (4.6) upon eliminating the parameter $x \in(0, x)$. 


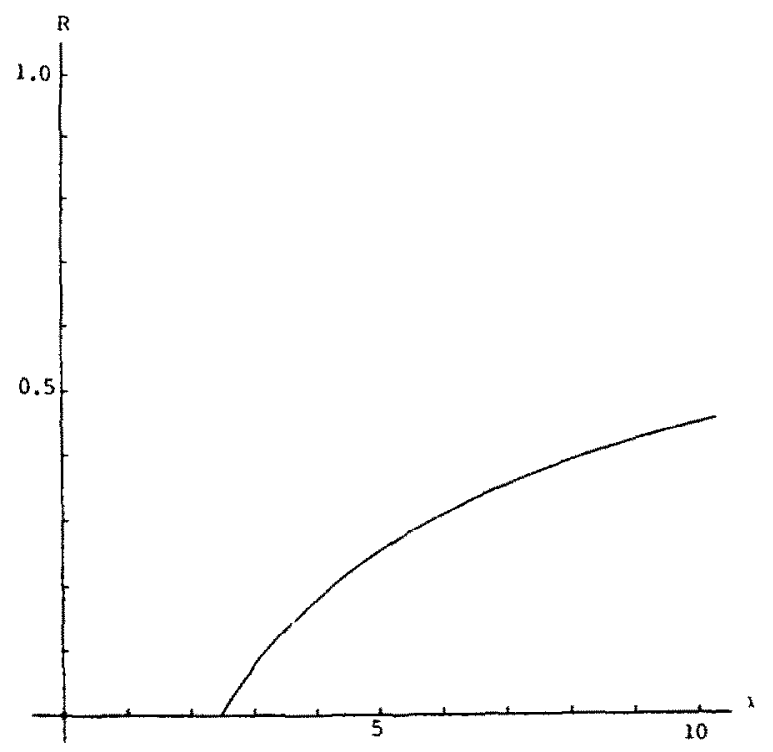

Fig. 2. See Fig. 1.

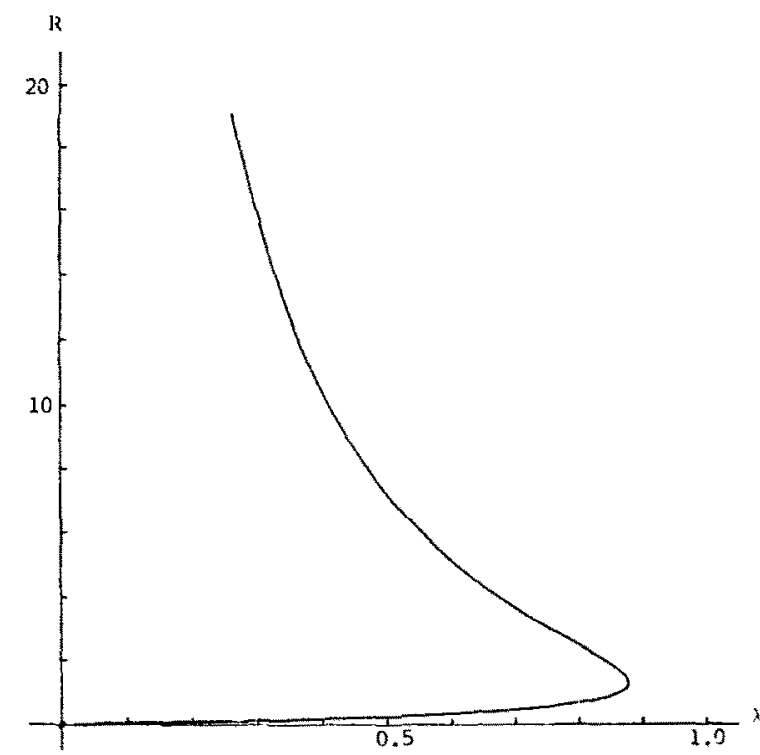

Fig. 3. See Fig. 1.

and we immediately find:

$$
\begin{aligned}
& \lambda(\alpha) \equiv \lambda_{1}=(\pi / 2)^{2}, \\
& R(\alpha)=\frac{1}{4} \alpha^{2}, h(R(\alpha))=\frac{1}{4} \lambda_{1} \alpha^{2} .
\end{aligned}
$$

Example 4.2. $V_{2}(x)=\frac{1}{4} x^{4}$. According to proposition 3.1, for this homogeneous potential we have

$$
\lambda(R)=\text { const. } R^{-t}
$$

Example 4.3. $V_{3}(x)=\frac{1}{2} x^{2}+\frac{1}{4} x^{4}$. For this potential $R^{*}=\infty$ and Lemma 2.12 applies. Note that there is no a priori bound for the solution: $\alpha \in(0, \infty)$, and

$$
R(\alpha) \rightarrow \infty, \lambda(\alpha) \downarrow 0 \text { for } \alpha \rightarrow \infty \text {. }
$$

The graph of $R$ vs $\lambda$ is obtained from (4.5) and (4.6) using numerical means: see Fig. 1. 
Example 4.4. $V_{4}(x)=1-\frac{1}{\cosh x}$. This potential is bounded, $R^{*}=1$, and Lemma 2.14 applies. Note that $\alpha \in(0, \infty)$ and

$$
R(\alpha) \uparrow 1, \lambda(\alpha) \rightarrow \infty \text { for } \alpha \rightarrow \infty .
$$

(Fig. 2).

Example 4.5. $V_{5}(x)=e^{|x|}-1$. This potential is not differentiable at $x=0$. As has already been remarked in the proof of Lemma 2.1, the normal mode corresponding to the solution of (2.3) according to (2.5) is only a $C^{1}$-function (there is a finite jump in the second derivative at time $t=\frac{1}{2} T$ ). For this potential the relation between $\lambda$ and $R$ is not unique: see Fig. 3 .

Remark 4.6. Note that the potentials $V_{3}$ and $V_{4}$ both have $x \rightarrow V_{1}(x)$ as linearization around $x=0$. Hence, considering the non-trivial solution of equation (2.3) as a bifurcated one from the trivial solution $x \equiv 0$ (which is a solution of (2.3) for any $\lambda \in R$ ), the bifurcation value of $\lambda$, i.e. $\lambda_{1}=(\pi / 2)^{2}$, is simply the smallest eigenvalue of the linearized eigenvalue problem, i.e. precisely the problem (2.3) with potential $V_{1}$. As is well known, for these potentials there are also solutions of equation (2.3) that are not sign-definite [these are the solutions that bifurcate from the trivial one at the other eigenvalues of the linearized eigenvalue problem; these solutions are saddle point like critical points of the variational problem (2.12)].

\section{Direct sum potentials}

For simplicity of exposition we shall restrict ourselves to two-dimensional systems. Writing $\left(x_{1}, x_{2}\right)=(x, y)$, we shall consider potentials of the form

$$
V(x, y)=U(x)+W(y)
$$

where $U$ and $W$ are positive multiples of any of the one-dimensional potentials $V_{i}$ considered above.

Among the various normal modes that a system with potential (4.8) may have, there are two families of special (simple) normal modes:

(i) an $x$-mode, for which $x \neq 0, y \equiv 0$,

(ii) an $y$-mode, for which $x \equiv 0, y \neq 0$.

In the following, quantities that are related to the $x$-mode ( $y$-mode) will be denoted by

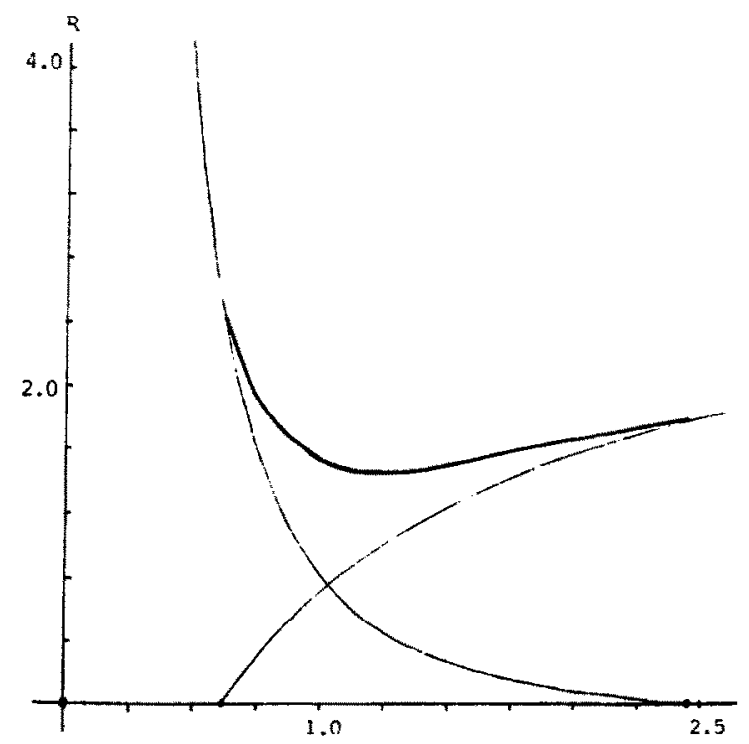

Fig. 4. Normal modes for the potential $V(x, y)=V_{3}(x)+4 V$ (y). Shown are the minimal $x$ - and $y$-mode, and the normal modes that can be composed from these. Observe that. for a given value of $R$. there may be $1,2,3$ or 4 of these normal modes; for a given value of $\lambda$ there may be one or three normal modes. 


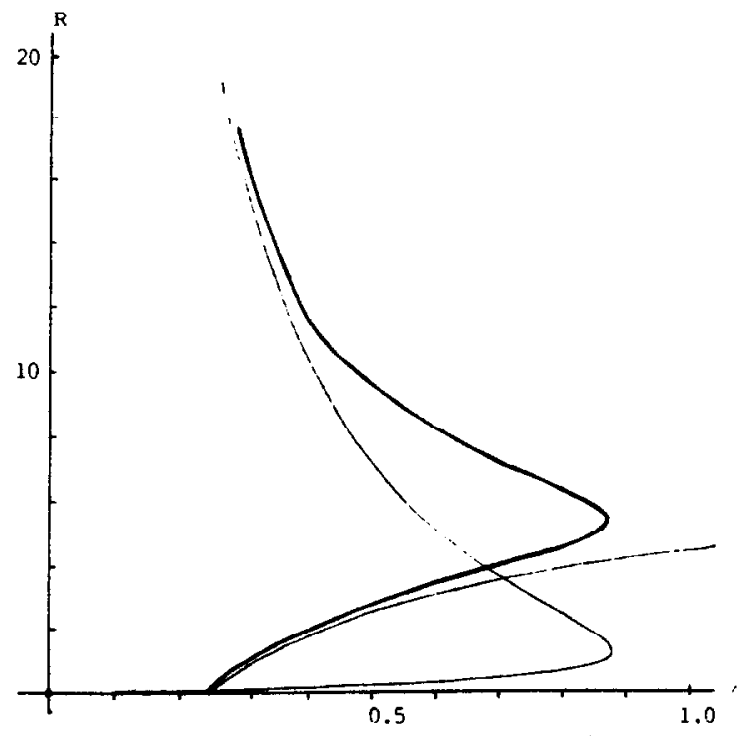

Fig. 5. Normal modes for the potential $V(x, y)=10 V_{4}(x)+V_{5}(y)$. Shown are the minimal $x$-mode, the $y$-mode and the modes composed from these.

Given $R>0$, there may be one, two or three normal modes; for a given value of $\lambda$ there may be one, two, three or five of these modes.

a subscript $U$ ( $W$ respectively); for instance: $\lambda_{v}(R), R_{W}^{*}, h_{U}(R)$, etc.

First observe that for any value of $R$ with

$$
0<R<\min \left(R_{U}^{*}, R_{W}^{*}\right)
$$

both the $x$-mode and the $y$-mode are possible solutions at this value of $R$. However, for specific values of $R$, there may be more normal modes. To see that, we consider in the first instant $x$-and $y$-modes that have the same value of $\lambda$. Indeed, if there exist values $R_{1}$ and $R_{2}$ such that

$$
\lambda_{v}\left(R_{1}\right)=\lambda_{w}\left(R_{2}\right)
$$

the $x$ - and $y$-mode at $R_{1}$ and $R_{2}$ respectively, may be "composed" to make a new normal mode for which $x \neq \equiv, y \neq 0$ and for which $R=R_{1}+R_{2}$. In this way it is possible, simply by "adding" the relevant $R$ vs $\lambda$ curves of the $x$ - and $y$-mode, to investigate the number of such normal modes (both at a given value of $R$, and also at a given value of $\lambda$ ). By way of example, Fig. 4 shows the normal modes that can be obtained in this way for the potential

$$
V(x, y)=V_{3}(x)+4 V_{4}(y)
$$

and Fig. 5 represents normal modes for the potential

$$
V(x, y)=10 V_{4}(x)+V_{5}(y)
$$

(with $V_{i}$ the functions considered before).

Remark 4.7. It is also possible to use the energy $E$ instead of $R$ as the bifurcation parameter. In that case, the results in the examples given above are qualitatively the same. But, using the parameter $R$, it is possible to make the variational characterization of the normal modes as given by (2.12) more precise. To see this, define a function $f_{R}$ in the following way:

$$
f_{R}(r):=h_{U}(R-r)+h_{W}(r) \text { for } 0 \leqslant r \leqslant R .
$$

[When dealing with bounded potentials $U$ (or $W$ ), we formally put $h_{U}(R)=\infty$ if $R \geqslant R_{U}^{*}$ (and $h_{W}$ analogously).] Then $f_{R}(0)=h_{U}(R)$, if finite, corresponds to the $x$-mode (i.e. $R_{U}=R$, $R_{W}=0$ ), whereas $f_{R}(R)=h_{W}(R)$, if finite, corresponds to the $y$-mode (i.e. $R_{U}=0, R_{W}=R$ ).

Furthermore, any critical point $\hat{r}$ of this function (if any)

$$
\frac{\mathrm{d}}{\mathrm{d} r} f_{R}(\hat{r})=0,0<\hat{r}<R,
$$


corresponds to a normal mode that can be composed as described above from an $x$-mode with $R_{U}=R-\hat{r}$ and $y$-mode with $R_{W}=\hat{r}$. This is immediate from the fact that condition (4.12) implies (4.10):

$$
\lambda_{v}(R-\hat{r})=\lambda_{w}(\hat{r})
$$

as follows from proposition 2.10.

Acknowledgements-It is a pleasure to thank P. van der Varst for several stimulating discussions during the preparation of this manuscript, and G. W. M. Staarink who performed the numerical operations that led to the figures presented in Section 4.

\section{REFERENCES}

1. T. L. Johnson and R. H. Rand, On the existence and bifurcation of minimal normal modes. Int. J. Non-Linear Mechanics 14, 1-12 (1979).

2. H. Seifert, Periodische Bewegungen mechanischer Systeme. Math. Z. 51, 197-216 (1948).

3. A. Weinstein, Periodic orbits for convex Hamiltonian systems. Ann. Math. 108, 507-518 (1978).

4. F. H. Clarke, Periodic solutions to Hamiltonian inclusions. J. diff. Eqs 40, 1-6 (1981).

5. P. H. Rabinowitz, Periodic solutions of Hamiltonian systems; Communs pure appl. Math. 31, 157-184 (1978).

6. F. H. Clarke and I. Ekeland, Hamiltonian trajectories having prescribed minimal period. Communs pure appl. Math. 33, 103-116 (1980).

7. A. Weinstein, Langrangian submanifolds and Hamiltonian systems. Ann. Math. 98, 377-410 (1973).

8. A. Weinstein, Normal modes for non-linear Hamiltonian systems. Inv. Math. 20, 47-\$7 (1973).

9. J. Moser, Periodic orbits near an equilibrium and a theorem by Alan Weinstein. Communs pure appl. Math. 29 727-747 (1976).

10. 1. Ekeland and J. M. Lasry, On the number of periodic trajectories for a Hamiltonian flow on a convex energy surface. Ann. Math. 112, 283-319 (1980).

11. P. H. Rabinowitz, On subharmonic solutions of Hamiltonian systems. Communs pure Appl. Math. 33, 609-633 (1980).

12. A. D. Ioffe and V. M. Tihomirov, Theory of Extremal Problems. North-Holland, Amsterdam (1979).

13. E. W. C. van Groesen, Dual and inverse formulations of constrained extremum problem. Math. Modelling 1, 237-254 (1980).

14. R. M. Rosenberg, Normal modes of nonlinear dual-mode systems. J. appl. Mech. 27 263-268 (1960).

15. R. M. Rosenberg, The normal modes of nonlinear n-degree of freedom systems. J. appl. Mech. 29 7-14 (1962).

16. L. A. Ljusternik and L. G. Schnirelmann, Topological methods in the Calculus of Variations. Hermann, Paris (1934).

17. P. H. Rabinowitz, Some aspects of non-linear eigenvalue problems, Rocky Mt. J. Math. 3, 161-202 (1973).

18. R. M. Rosenberg. The Ateb functions and their properties, Qt. appl. Math. 21, 37 (1963).

19. P. van der Varst, On normal mode vibrations of nonlinear conservative systems. Ph.D. Thesis Eindhoven University of Technology, (to appear).

\section{Zusammenfassung:}

Normale Schwingungsformen gerader Hamiltonscher Systeme klassischer Art werden als kritische Punkte eines normalisierten Funktionals der kinetischen Energie auf ebenen Gruppen des Funktionals der potentiellen Energie charakterisiert. Mit Hilfe dieser eingeschraenkten Variationsformulierung wird das Vorhandensein von mindestens einer Familie normaler Schwingungsformen bewiesen und fuer eine begrebzte Kasse von Potentialen wird die Verzweigung von Schwingungsformen untersucht. Weiterhin wird eine Vermitung bezueglich einer unteren Begrenzung der Anzahl von normalen Schwingungsformen falls das Potential homogen ist bewiesen.

\section{Résumé:}

On caractérise les modes normaux de systèmes Hamiltoniens paires et de type classique comme points critiques d'une fonctionnelle d'énergie cinétique normalisée sur des ensembles de niveaus de la fonctionnelle d'énergie potentielle. A l'aide de cette formulation variationnelle forcée, on prouve l'existence d'au moins une famille de modes normaux et pour un ensemble limité de potentiels, on examine la séparation de modes. De plus on démontre une supposition sur une limite inférieure du nombre de modes normaux dans le cas où le potentiel est homogène. 